JOURNAL OF

SYMPLECTIC GEOMETRY

Volume 5, Number 1, 9-18, 2007

\title{
EFFECTIVE CLASSES AND LAGRANGIAN TORI IN SYMPLECTIC FOUR-MANIFOLDS
}

\author{
JEAN-YVES WELSCHINGER
}

\begin{abstract}
An effective class in a closed symplectic four-manifold is a twodimensional homology class which is realized by a $J$-holomorphic cycle for every tamed almost complex structure $J$. We first prove that effective classes are orthogonal to Lagrangian tori with respect to the intersection form. We then deduce an invariant under birational transformations of closed symplectic four-manifolds. We finally prove using the same techniques of symplectic field theory that the unit cotangent bundle of a compact orientable hyperbolic Lagrangian surface does not embed as a hypersurface of contact type in a rational or ruled symplectic four-manifold.
\end{abstract}

Dedicated to Dusa McDuff

\section{Statement of the main results}

Let $(X, \omega)$ be a closed symplectic 4-manifold. A two-dimensional homology class $d \in H_{2}(X ; \mathbb{Z})$ is called effective when it is realized by a $J$-holomorphic two-cycle for every almost complex structure $J$ tamed by the symplectic form $\omega$. Denote by $B_{\omega}(X)$ the subgroup of $H_{2}(X ; \mathbb{Z})$ generated by effective classes. Likewise, denote by $A_{\omega}^{1}(X)$ (resp. $\left.A_{\omega}^{2}(X)\right)$ the subgroup of $H_{2}(X ; \mathbb{Z})$ generated by Lagrangian tori (resp. orientable hyperbolic Lagrangian surfaces).

Theorem 1.1. For every closed symplectic 4-manifold $(X, \omega)$, the groups $A_{\omega}^{1}(X)$ and $B_{\omega}(X)$ are orthogonal to each other with respect to the intersection form.

(see Remark 6 of Section 2.1 for a similar result with $A_{\omega}^{2}(X)$ ). Recall that a pseudo-holomorphic sphere in a closed symplectic 4-manifold is a sphere which can be made $J$-holomorphic for some tamed almost complex structure $J$. The Chern number of such a sphere is the evaluation of the first Chern class of the manifold on its fundamental class. 
Corollary 1.2. Let $L$ be a smooth Lagrangian torus and $S$ be a pseudoholomorphic sphere of positive Chern number in a closed symplectic 4-manifold. Then, $L$ and $S$ have vanishing intersection index.

Proof. Blowing up the symplectic manifold finitely many times outside $L$ if necessary, we may assume that $S$ has Chern number 1. From Lemma 3.1 of $[\mathbf{1 1}]$, we know that Chern number 1 pseudo-holomorphic spheres define effective classes. The result thus follows from Theorem 1.1.

Note that from Theorem 1.4 of [13], pseudo-holomorphic spheres having Chern number greater than one only exist in rational or ruled symplectic 4-manifolds, where Lagrangian tori actually vanish in homology, see Example 1 below. Note also that embedded Chern number 1 pseudo-holomorphic spheres correspond from adjunction formula to embedded symplectic $(-1)$ spheres. Corollary 1.2 suggests that after some symplectic isotopy of $S$, the possible intersections between $S$ and $L$ can be removed. This actually holds true, as follows from Theorem 1.3.

Theorem 1.3. Let $L$ be a smooth orientable Lagrangian surface of positive genus and $S$ be a pseudo-holomorphic sphere of positive Chern number in a closed symplectic 4-manifold. Then, $S$ is symplectically isotopic to a surface disjoint from $L$.

Recall that two closed symplectic 4-manifolds are said to be birational to each other if one can pass from one to another through a finite sequence of blow-ups and down of symplectic balls, see $[\mathbf{7}, \mathbf{1 2}]$.

Corollary 1.4. The group $H_{2}(X ; \mathbb{Z}) / B_{\omega}(X)$ is invariant under birational transformations of the closed symplectic 4-manifold $(X, \omega)$. Let $(\tilde{X}, \tilde{\omega})$ be a blow-up of $(X, \omega)$; then the groups $A_{\tilde{\omega}}^{1}(\widetilde{X}), A_{\tilde{\omega}}^{2}(\widetilde{X})$ are subgroups of $A_{\omega}^{1}(X)$, $A_{\omega}^{2}(X)$, respectively.

The groups $A_{\omega}^{1}(X), A_{\omega}^{2}(X)$ may actually be invariant under birational transformations of $(X, \omega)$ as well. This would follow from a positive solution to the following problem.

Problem 1.5. Given a closed symplectic ball $B$ and a smooth compact orientable Lagrangian surface $L$ of positive genus in a closed symplectic 4-manifold. Is this Lagrangian surface homologous to a smooth homeomorphic Lagrangian surface disjoint from $B$ ?

I initially believed a Hamiltonian isotopic surface would be disjoint from $B$ and am grateful to Paul Biran for pointing out to me it was already not the case for the Clifford torus in the complex projective plane. Paul Biran also proved in Theorem 1.B of [2] that any closed symplectic ball of radius $\lambda$ in the complex projective plane, $\lambda^{2} \geq \frac{1}{2}$, should intersect the real projective plane. 
Note that $A_{\omega}^{1}(X), A_{\omega}^{2}(X)$ are not invariant under deformation of the symplectic form $\omega$. The orthogonal of a class $[\omega]$ of $H^{2}(X ; \mathbb{R})$ indeed only intersects $H_{2}(X ; \mathbb{Z})$ at the origin in general. Likewise, $B_{\omega}(X)$ is not a priori designed to be invariant under deformation of $\omega$. Here there is a confusing analogy with the Hodge decomposition of complex surfaces, see Theorem $(2.10)$ of $[\mathbf{1}]$. Namely, the groups $\left(H^{2,0}(X ; \mathbb{C}) \oplus H^{0,2}(X ; \mathbb{C})\right) \cap H^{2}(X ; \mathbb{Z})$ and $H^{2}(X ; \mathbb{Z}) / H^{1,1}(X ; \mathbb{Z})$ are invariant under birational transformations of the complex structure of the surface, but not under deformation of it. Following this analogy and given a closed symplectic 4 -manifold $(X, \omega)$, do there exist natural subspaces of $H_{2}(X ; \mathbb{C})$ whose trace on $H_{2}(X ; \mathbb{Z})$ are $A_{\omega}(X)$ and $B_{\omega}(X) ?$

\section{Proof of the main results}

Let $L$ be a torus equipped with a flat metric, $S^{*} L$ be its unit cotangent bundle and $\pi: S^{*} L \rightarrow L$ the canonical projection. The manifold $S^{*} L$ is equipped with a canonical contact form $\lambda$, namely the restriction of the Liouville one-form of its cotangent bundle. We denote by $R_{\lambda}$ the subgroup of $H_{1}\left(S^{*} L ; \mathbb{Z}\right)$ generated by its closed Reeb orbits.

Lemma 2.1. The restriction of $\pi_{*}: H_{1}\left(S^{*} L ; \mathbb{Z}\right) \rightarrow H_{1}(L ; \mathbb{Z})$ to $R_{\lambda}$ is an isomorphism.

Proof. The Reeb flow on $S^{*} L$ coincides with the geodesic flow. Closed Reeb orbits are thus the lifts of closed geodesics on $L$. Now $S^{*} L$ is diffeomorphic to a product of $L$ with the sphere $S$ of directions in $L$, and $\pi$ is the projection onto the first factor. Since geodesics of $L$ have a constant direction, the projection onto the second factor maps every Reeb orbit to a point of $S$. From the Künneth formula, we get the isomorphism $H_{1}\left(S^{*} L ; \mathbb{Z}\right) \cong H_{1}(L ; \mathbb{Z}) \times H_{1}(S ; \mathbb{Z})$ and, from what we have just noticed, that this isomorphism maps $R_{\lambda}$ into $H_{1}(L ; \mathbb{Z}) \times\{0\}$. Since generators of $H_{1}(L ; \mathbb{Z})$ can be realized by closed geodesics, the latter map is onto. Since $\pi_{*}$ is the projection onto the first factor, it is an isomorphism once restricted to $R_{\lambda}$.

Proof of Theorem 1.1. Let $L$ be a Lagrangian torus and $d$ be an effective class. Following the principle of symplectic field theory [6], we stretch the neck of the symplectic manifold in the neighbourhood of $L$ until the manifold splits in two parts, one part being the cotangent bundle of the torus and the other being $X \backslash L$. We produce this splitting in such a way that both parts have the contact manifold $\left(S^{*} L, \lambda\right)$ at infinity. Let $J_{\infty}$ be a $C R$ structure on this contact 3-manifold, which we extend to an almost complex structure $J$ with cylindrical end on both parts $T^{*} L$ and $X \backslash L$. The latter is the limit of a sequence $J_{n}$ of almost complex structures of $(X, \omega)$. Since $d$ is effective, we may associate a sequence $C_{n}$ of $J_{n}$-holomorphic two-cycles 
homologous to $d$. From the compactness Theorem in SFT [4], we extract a subsequence converging to a broken $J$-holomorphic curve $C$, which we assume for convenience to have only two levels-the general case follows easily from this one. Denote by $C^{L}$ the part of $C$ in $T^{*} L$ and by $C^{X}$ the part in $X \backslash L$. Both curves $C^{L}$ and $C^{X}$ have cylindrical ends asymptotic to the same set of closed Reeb orbits with same multiplicities. Let $C_{1}^{L}, \ldots, C_{k}^{L}$ denote the irreducible components of $C^{L}$ and $R_{1}, \ldots, R_{k}$ be the corresponding sets of closed Reeb orbits. These sets $R_{1}, \ldots, R_{k}$ define integral one-cycles in $S^{*} L$ and we denote by $\left[R_{1}\right], \ldots,\left[R_{k}\right]$ their homology classes. These onecycles are boundaries of the two-chains $C_{1}^{L}, \ldots, C_{k}^{L}$ in $T^{*} L$, so that with the notation of Lemma $2.1, \pi_{*}\left(\left[R_{i}\right]\right)$ vanishes for every $i \in\{1, \ldots, k\}$. Since $\left[R_{1}\right], \ldots,\left[R_{k}\right]$ belong to the subgroup $R_{\lambda}$, we deduce from Lemma 2.1 that $\left[R_{1}\right], \ldots,\left[R_{k}\right]$ actually vanish. Let $S_{1}, \ldots, S_{k}$ be integral two-chains of $S^{*} L$ having $R_{1}, \ldots, R_{k}$ as boundaries, and $S$ be the sum of these $k$ chains. Then, $C^{L}-S$ is an integral two-cycle contained in $T^{*} L, C^{X}+S$ is an integral two-cycle contained in $X \backslash L$ and the sum of these cycles is homologous to $d$. Now, $L$ and $C^{X}+S$ are disjoint from each other and the second homology group of $T^{*} L$ is generated by $[L]$ itself. Since the latter has vanishing self-intersection, we deduce that the intersection index of $L$ and $C^{L}-S$ vanishes. As a consequence, the intersection index of $d$ and $[L]$ vanishes. Since this holds for any Lagrangian torus or effective class, Theorem 1.1 is proved.

Proof of Theorem 1.3. Let us denote the manifold by $(X, \omega)$ and equip it with a tamed almost complex structure $J_{0}$ making $S$ holomorphic. Blowing up $(X, \omega)$ finitely many times if necessary, or rather requiring that $S$ pass through finitely many points, we may assume that $S$ has Chern number 1 . We then equip $L$ with a metric of constant curvature and stretch the neck of $J_{0}$ in a neighbourhood of $L$. This stretching produces a family $\left(J_{t}\right)_{t \in \mathbb{R}_{+}}$ of tamed almost complex structures together with an isotopy $\left(S_{t}\right)_{t \in \mathbb{R}_{+}}$of $J_{t}$-holomorphic spheres, where $S_{0}=S$, see Lemma 3.1 of [11]. We claim that as soon as the neck is sufficiently long, these $J_{t}$-holomorphic curves $S_{t}$ are disjoint from $L$. From the compactness Theorem in SFT [4], we would indeed otherwise be able to extract a subsequence converging to a two-levels rational curve having a non-empty level $C^{L}$ in $T^{*} L$ and a nonempty level $C^{X}$ in $X \backslash L$. Since $L$ does not have any contractible closed geodesic, no component of $C^{L}$ can be planar. We deduce that $C^{X}$ contains at least two $J$-holomorphic planes, every tree containing at least two leaves. Let us equip the tangent bundle of $T^{*} L$ with its canonical trivialization along closed Reeb orbits, namely the one tangent to the Lagrangian fibers of $T^{*} L$. The obstruction to extend this trivialization over the whole $C^{L}$ then vanishes since the tangent bundle of $T^{*} L$ is the complexification of an orientable Lagrangian bundle. 
Let us assume first that $L$ is hyperbolic. The Conley-Zehnder index of every closed Reeb orbit then vanishes in the chosen trivialization, see Proposition 1.7.3 of [6]. Denote by $\mu_{j}$ twice the obstruction to extend this trivialization over the component $C_{j}^{X}$ of $C^{X}$. From Theorem 2.8 of [8], we deduce that the virtual dimension of the space of deformations of $C_{j}^{X}$ is given as $\mu_{j}-\chi_{j}$, where $\chi_{j}$ denotes the Euler characteristic of $C_{j}^{X}$. In particular, this dimension can only increase under branched coverings. The genericity of $J$ then guaranties the inequality $\mu_{j}-\chi_{j} \geq 0$. If $C_{j}^{X}$ is a plane, this virtual dimension is odd so that actually $\mu_{j}-\chi_{j} \geq 1$. Since $C^{X}$ contains at least two planes and since the Euler characteristic of $C^{L}$ is not positive, we finally deduce after summation that the tangent Maslov index of $S$ is bounded from below by its Euler characteristic plus two, a contradiction.

The proof goes along the same lines when $L$ is a torus. The Reeb flow is then degenerated and closed Reeb orbits come in one-parameter families. The Fredholm theory in this framework has been studied by Frédéric Bourgeois in his thesis. The upshot in our case is that, once more, the virtual dimensions of the spaces of deformations of the curves $C_{j}^{X}$ write $\mu_{j}-\chi_{j}$. This means that these dimensions behave as if the Reeb flow would be non-degenerated with vanishing Conley-Zehnder indices. The reason for this is that these Reeb orbits rather have - after small perturbationConley-Zehnder index 1 contributing as -1 to the virtual dimension, but this contribution gets compensated by the freedom these orbits have to move in their one-parameter families, see Sections 5.1, 5.2 and 9.4 of [3]. Hence the result.

Proof of Corollary 1.4. It suffices to prove the invariance under one blow-up, say the blow-up $(\tilde{X}, \tilde{\omega})$ of a closed symplectic ball $B$ of $(X, \omega)$. The group $H_{2}(X ; \mathbb{Z})$ is canonically isomorphic to the orthogonal of the exceptional sphere $S$ in $H_{2}(\widetilde{X} ; \mathbb{Z})$. We claim that this isomorphism sends effective classes to effective classes and classes realized by orientable surfaces of positive genus in $\widetilde{X}$ to classes realized by orientable surfaces of positive genus in $X$. Indeed, let $\widetilde{L}$ be an orientable surface of positive genus of $\widetilde{X}$. Theorem 1.3 provides an isotopy $S_{t}$ of embedded symplectic (-1)-spheres such that $S_{0}=S$ and $S_{1}$ is disjoint from $\widetilde{L}$. Moser stability for pairs implies that this isotopy $S_{t}$ is actually induced by an isotopy of symplectic diffeomorphisms of the ambient manifold $(\widetilde{X}, \tilde{\omega})$, see Corollary 4.1.B of $[\mathbf{1 4}]$. The corresponding family of inverse diffeomorphisms makes it possible to isotop $\widetilde{L}$ to a surface disjoint from $S$. Blowing down the ball $B$ does not affect the latter. The second part of Corollary 1.4 is thus proved.

Now, let $\tilde{d}$ be an effective class of $(\tilde{X}, \tilde{\omega})$. Since $[S]$ is itself effective, we can assume $\tilde{d}$ to be orthogonal to $[S]$ and denote by $d$ the corresponding class of $H_{2}(X ; \mathbb{Z})$. Let $J$ be an almost complex structure of $X$ tamed by $\omega$ and 
$x \in B$. There exists a sequence $\left(J_{n}\right)_{n \in \mathbb{N}}$ of tamed almost complex structures integrable in a neighborhood of $x$ which converge uniformly to $J$. This sequence can actually be constructed for $n$ large enough by interpolation of the constant almost complex structure $\left.J\right|_{x}$ in a Darboux ball of radius $\frac{1}{n}$ around $x$ and the structure $J$ outside a Darboux ball of radius $\frac{2}{n}$ around $x$. For every $n$, the complex blow-up $\widetilde{J}_{n}$ of $J_{n}$ at $x$ is tamed by $\tilde{\omega}$. Indeed, we know from $[\mathbf{7}, \mathbf{1 2}]$ that $B$ is contained in a larger open ball $B_{\epsilon}$. The symplectic form $\omega$ on $B_{\epsilon} \backslash\{0\}$ reads $d\left(\mathrm{e}^{t} \lambda\right)$, where $t<\rho_{\epsilon}$ and $\lambda$ is the standard contact form of the unit sphere, whereas the symplectic form $\tilde{\omega}$ is rather the symplectic form $\omega$ of $B_{\epsilon} \backslash B$ which reads $d\left(\mathrm{e}^{h(t)} \lambda\right)$, where $\left.h:\right]-\infty, \rho_{\epsilon}[\rightarrow] \rho_{B}, \rho_{\epsilon}[$ and $h^{\prime}(t)>0$. The spaces of almost complex structures tamed by these two forms are exactly the same, since they coincide up to a positive factor on every sphere and on every kernel of $d \lambda$. This complex blow-up $\widetilde{J}_{n}$ of $J_{n}$ comes with a $\widetilde{J}_{n}$-holomorphic map $\pi_{n}$ onto $\left(X, J_{n}\right)$. By assumption, there exists a $\widetilde{J}_{n}$-holomorphic curve $\widetilde{C}_{n}$ homologous to $\tilde{d}$. The image $C_{n}=\pi_{n}\left(\widetilde{C}_{n}\right)$ is a $J_{n}$-holomorphic curve homologous to $d$. Now from Gromov compactness Theorem, there exists a subsequence of $C_{n}$ converging to a $J$-holomorphic curve $C$ homologous to $d$ (see Theorem 1 of $[\mathbf{9}]$ for the $C^{0}$ version of Gromov compactness theorem we are using here). As a consequence, $d$ is effective.

Conversely, assume that $d$ is an effective class of $(X, \omega)$, we have to prove that the corresponding class $\tilde{d}$ orthogonal to $[S]$ in $H_{2}(\widetilde{X} ; \mathbb{Z})$ is effective as well. Let $\widetilde{J}$ be an almost complex structure of $\widetilde{X}$ tamed by $\tilde{\omega}$. We can assume from Gromov compactness theorem that $\widetilde{X}$ contains no $\widetilde{J}$ holomorphic sphere of non-positive Chern number, since this is a generic condition. The manifold $\widetilde{X}$ contains then a unique embedded $\widetilde{J}$-holomorphic sphere $\widetilde{S}$ homologous to $S$. The normal bundle of $\widetilde{S}$ has the structure of a complex line bundle and a neighbourhood of its zero section is identified with a neighbourhood of $\widetilde{S}$ in $\widetilde{X}$. Equip this normal bundle with the structure of a holomorphic line bundle $N$. There exists a sequence $\left(\widetilde{J}_{n}\right)_{n \in \mathbb{N}}$ of tamed almost complex structures integrable in a neighbourhood of $\widetilde{S}$ in $\widetilde{X}$ which convege uniformly to $\widetilde{J}$. The latter can actually be constructed for $n$ large enough by interpolation of the holomorphic structure of $N$ in a neighbourhood of size $\frac{1}{n}$ of the zero section and the structure $\widetilde{J}$ outside a neighbourhood of size $\frac{2}{n}$ of the zero section. For every $n \in \mathbb{N}$, the complex blow down of $\widetilde{S}$ to a point $x \in X$, possible thanks to Grauert's contractibility criterion (see Theorem 2.1 of $[\mathbf{1}]$ ), produces an almost complex structure $J_{n}$ of $X$ together with a $\widetilde{J}_{n}$-holomorphic map $\pi_{n}$ onto $\left(X, J_{n}\right)$. The structure $J_{n}$ is tamed by $\omega$ since as before the symplectic forms on the annulus $d\left(\mathrm{e}^{t} \lambda\right), t<\rho_{\epsilon}$ and $\left.d\left(\mathrm{e}^{h(t)} \lambda\right), h:\right]-\infty, \rho_{\epsilon}[\rightarrow] \rho_{B}, \rho_{\epsilon}\left[\right.$ and $h^{\prime}(t)>0$ tame the same almost complex structures. Now since $d$ is effective, there exists a $J_{n}$-holomorphic curve $C_{n}$ homologous to $d$. Perturbing $J_{n}$ away from $x$ if 
necessary, we may assume that $C_{n}$ does not contain $x$. The strict transform of $C_{n}$ then produces a $\widetilde{J}_{n}$-holomorphic curve $\widetilde{C}_{n}$ homologous to $\tilde{d}$. Again, from Gromov compactness theorem, there exists a subsequence of $\widetilde{C}_{n}$ converging to a $\widetilde{J}$-holomorphic curve $\widetilde{C}$ homologous to $\tilde{d}$ (see Theorem 1 of [9] for the $C^{0}$ version of Gromov compactness theorem we are using here). As a consequence, $\tilde{d}$ is effective.

2.1. Remarks. 1) We have actually proved more than claimed in Theorem 1.1 , namely for a class $d \in H_{2}(X ; \mathbb{Z})$ to be orthogonal to a Lagrangian torus $[L]$, it suffices that $d$ be realized by a sequence of $J_{n}$-holomorphic two-cycles, for a sequence $J_{n}$ having a flat neck stretching to infinity.

2) From the results of Taubes [16], any Seiberg-Witten basic class is effective and thus, from Theorem 1.1, we deduce that SW-basic classes are orthogonal to Lagrangian tori. This fact was already known, it indeed follows from the adjunction inequality $[\mathbf{1 0}, \mathbf{1 5}]$. Our space $B_{\omega}(X)$ might however be bigger than the one generated by SW-basic classes? Also, our proof remains in the symplectic category and offers possibilities to have counterparts in higher dimensions.

$3)$ If $(X, \omega)$ is Kähler, then the Poincaré dual of $B_{\omega}(X)$ is contained in the intersection of $H^{1,1}(X ; \mathbb{Z})$ on every complex structure of $X$ tamed by $\omega$. How smaller can it be?

4) From the Hodge Index Theorem, it follows that the intersection $A_{\omega}^{1}(X) \cap B_{\omega}(X)$ is torsion for every Kähler surface. This intersection indeed lies in the isotropic cone of the Lorentzian $H^{1,1}(X ; \mathbb{R})$ and is orthogonal to the symplectic form which lies in the positive cone (compare Example 1 of Section 2.2). I do not see at the moment whether or not this holds for every closed symplectic 4-manifold (and am grateful to Stéphane Lamy for raising the question to me). More generally, one may wonder whether the intersection form restricted to $B_{\omega}(X)$ has to be non-degenerated (e.g., of signature $(1, n)$ when non-vanishing). This is the case at least for rational surfaces from Example 2 of Section 2.2 and for Kähler surfaces with $b_{2}^{+} \geq 2$ and $K_{X}^{2}>0$ since from Taubes' results $[\mathbf{1 6}], B_{\omega}(X)$ contains the canonical class $K_{X}$.

5) We made a crucial use of a property of the contact manifold $\left(S^{*} L, \lambda\right)$, namely that the subgroup $R_{\lambda}$ generated by its closed Reeb orbits has a rather big index in $H_{1}\left(S^{*} L ; \mathbb{Z}\right)$. We may then more generally wonder, given a contact manifold, how small can this subgroup $R_{\lambda}$ of "effective" homology classes be?

6) Using the same methods, one can prove an analog of Theorem 1.1 for orientable hyperbolic Lagrangian surfaces. Namely, if $L$ is such a surface and $d$ is a class having a non-trivial Gromov-Witten invariant computed with differential forms vanishing along $L$, then $d$ and $L$ are orthogonal to each other in $H_{2}(X ; \mathbb{Z} / 2 \mathbb{Z})$. 
2.2. Examples. 1) If $(X, \omega)$ has a Lorentzian intersection form of signature $(1, n)$, then $A_{\omega}^{1}(X)$ is torsion and $A_{\omega}^{2}(X)$ vanishes. Indeed, every Lagrangian torus should be in the isotropic cone of the intersection form and should be orthogonal to the class of the symplectic form which lies in the positive cone. Likewise, every orientable hyperbolic surface has positive self-intersection and is orthogonal to $\omega$ in $H_{2}(X ; \mathbb{R})$. This last observation actually goes back to Comessatti, who made it in the framework of real algebraic geometry, namely for real components of a smooth real rational surface, see [5]. We can prove the following stronger statement.

Theorem 2.2. The unit cotangent bundle of an orientable hyperbolic Lagrangian surface does not embed as a hypersurface of contact type in a rational or ruled symplectic 4-manifold.

One may wonder whether such a unit cotangent bundle embeds at all in a rational or ruled 4-manifold. Note that we will actually prove that such a unit cotangent bundle and a pseudo-holomorphic sphere $S$ of Chern number $c_{1}(X)[S]$ greater than one cannot both exist in the same symplectic 4-manifold. However, Dusa McDuff already proved in Theorem 1.4 of [13] that the latter only exists in rational or ruled symplectic 4-manifold.

Proof. Assume that the rational or ruled symplectic 4-manifold $(X, \omega)$ contains a hypersurface of contact type contactomorphic to the unit cotangent bundle $\left(S^{*} L, \lambda\right)$ of an orientable hyperbolic Lagrangian surface $L$. Let us fix a neighbourhood of $\left(S^{*} L, \lambda\right)$ in $(X, \omega)$ symplectomorphic to a piece of the symplectization $\left(\mathbb{R} \times S^{*} L, d\left(\mathrm{e}^{t} \lambda\right)\right)$ and choose a point $x$ in the negative part of this Liouville neighbourhood, that is, in the past of $\left(S^{*} L, \lambda\right)$ with respect to the Liouville flow. Since $(X, \omega)$ is rational or ruled, it contains a pseudoholomorphic sphere $S$ passing through $x$ of Chern number $c_{1}(S)$ greater than one. We can now repeat the proof of Theorem 1.3. We choose a tamed almost complex structure $J_{0}$ on $X$ which is cylindrical over a $C R$-structure on $\left(S^{*} L, \lambda\right)$ in its chosen Liouville neighbourhood. We may assume that $S$ is $J_{0}$-holomorphic and fix $c_{1}(S)-2$ extra points $y$ on $S$. We then stretch the neck of $J_{0}$ in the chosen Liouville neighbourhood until the splitting in two parts $\left(X^{+} \backslash S^{*} L, J^{+}\right)$and $\left(X^{-} \backslash S^{*} L, J^{-}\right)$or one part $\left(X \backslash S^{*} L, J\right)$ depending on whether $X \backslash S^{*} L$ is connected or not. The $J_{0}$-holomorphic sphere deforms along the stretching until it splits into a several-level curve passing through $x$ and $y$. This curve in the limit cannot indeed be compact in $\left(X \backslash S^{*} L, \omega\right)$ since it passes through $x$ and in the Liouville neighbourhood, the map $(t, x) \in \mathbb{R} \times S^{*} L \mapsto \mathrm{e}^{t}$ induces a sub-harmonic function which thus cannot reach a maximum. Indeed, $d d^{\mathbb{C}} \mathrm{e}^{t}=d\left(\mathrm{e}^{t} d t \circ J\right)=-d\left(\mathrm{e}^{t} \lambda\right)$. For this reason, every curve passing through $x \in X$ has to cross $\left(S^{*} L, \lambda\right)$. Now again as in the proof of Theorem 1.3, when $J_{0}$ is generic enough, every connected component of this several-levels $J^{ \pm}$-holomorphic curve has to be rigid together with its incidence conditions since its degree of freedom has to be 
non-negative and the sum of these degrees over all components vanishes. However, two of these components have to be planar and the virtual dimension given by Theorem 2.8 of [8] for such planar components is odd, a contradiction.

2) If $(X, \omega)$ is a blow-up of the projective plane, then $B_{\omega}(X)=H_{2}(X ; \mathbb{Z})$. Indeed, exceptional divisors are effective classes, and the strict transform of a line has non-trivial GW-invariants.

$3)$ If $X$ is a product of two curves $\left(C_{1}, \omega_{1}\right)$ and $\left(C_{2}, \omega_{2}\right)$ with symplectic form $\omega_{1} \ominus \omega_{2}$, then $A_{\omega}^{1}(X)$ contains the index two subgroup $H_{1}\left(C_{1} ; \mathbb{Z}\right) \otimes$ $H_{1}\left(C_{2} ; \mathbb{Z}\right)$ given by the Künneth formula. When, in addition, $\left(C_{1}, \omega_{1}\right)$ and $\left(C_{2}, \omega_{2}\right)$ are symplectomorphic tori, $A_{\omega}^{1}(X)$ contains the graph of the symplectomorphism. Note that $A_{\omega}^{1}(X)$ cannot have codimension less than one, since it lies in the orthogonal of the symplectic form.

4) If $X$ is a product of two genus 1 curves, then $B_{\omega}(X)$ vanishes, since for a generic complex structure, $H^{1,1}(X ; \mathbb{Z})$ vanishes. If instead one of the curve is not elliptic, then we know from Taubes' results $[\mathbf{1 6}]$ that $B_{\omega}(X)$ contains the canonical class of $X$.

\section{References}

[1] W. Barth, C. Peters and A. Van de Ven, Compact complex surfaces, Ergebnisse der Mathematik und ihrer Grenzgebiete (3), 4, SpringerVerlag, Berlin, 1984.

[2] P. Biran, Lagrangian barriers and symplectic embeddings, Geom. Funct. Anal. 11 (2001), (3), 407-464.

[3] F. Bourgeois, A Morse-Bott approach to Contact Homology, PhD dissertation, Stanford University, 2002.

[4] F. Bourgeois, Y. Eliashberg, H. Hofer, K. Wysocki and E. Zehnder, Compactness results in symplectic field theory, Geom. Topol., 7 (2003), 799-888.

[5] A. Comessatti, Sur la connessione delle superficie razionali reale, Ann. Math. 23 (1914), 215-285.

[6] Y. Eliashberg, A. Givental and H. Hofer, Introduction to symplectic field theory, GAFA 2000 (Tel Aviv, 1999), Geom. Funct. Anal., 2000, Special Volume, Part II, 560-673.

[7] V. Guillemin and S. Sternberg, Birational equivalence in the symplectic category, Invent. Math. 97 (1989), (3), 485-522.

[8] H. Hofer, K. Wysocki and E. Zehnder, Properties of pseudoholomorphic curves in symplectizations. III: Fredholm theory. Topics in nonlinear analysis, Nonlinear Differential Equations Appl., 35, Birkhäuser, Basel, 1999, 381-475. 
[9] S. Ivashkovich and V. Shevchishin, Gromov compactness theorem for $J$-complex curves with boundary, Internat. Math. Res. Notices (2000), (22), 1167-1206.

[10] P.B. Kronheimer and T.S. Mrowka, The genus of embedded surfaces in the projective plane, Math. Res. Lett. 1 (1994), (6), 797-808.

[11] D. McDuff, The structure of rational and ruled symplectic 4-manifolds, J. Amer. Math. Soc. 3 (1990), (3), 679-712.

[12] D. McDuff, Blow ups and symplectic embeddings in dimension 4, Topology 30 (1991), (3), 409-421.

[13] D. McDuff, Immersed spheres in symplectic 4-manifolds, Ann. Inst. Fourier (Grenoble) 42 (1992), (1-2), 369-392.

[14] D. McDuff and L. Polterovich, Symplectic packings and algebraic geometry. With an appendix by Yael Karshon. Invent. Math. 115 (1994), (3), 405-434.

[15] J.W. Morgan, Z. Szabó and C.H. Taubes, A product formula for the Seiberg-Witten invariants and the generalized Thom conjecture, J. Diff. Geom. 44 (1996), (4), 706-788.

[16] C.H. Taubes, Seiberg Witten and Gromov invariants for symplectic 4-manifolds (Ed. Richard Wentworth), First International Press Lecture Series, 2, International Press, Somerville, MA, 2000.

Unité DE MATHÉMATIQUeS PURES ET APPLiquÉES DE L'ÉCOLE NORMALE SUPÉRIEURE DE Lyon, CNRS — Université de Lyon 69364 Lyon CEDEX 07A, France

E-mail address: jean-yves.welschinger@umpa.ens-lyon.fr

Received 12/22/2006, accepted 03/02/2007.

I am grateful to the French Agence Nationale de la Recherche for its support. I wish also to acknowledge Paul Biran and Jean-Claude Sikorav for their fruitful remarks and interest on this work. 